\title{
Transthoracic Doppler echocardiography of the left internal mammary artery graft
}

\author{
Andreea Pârv ${ }^{1}$ Camelia Ober ${ }^{2}$, Dan Bindea ${ }^{3}$, Caius Duncea ${ }^{1}$
}

${ }^{1}$ Cardiology Department, Medical V Hospital, ${ }^{2}$ Cardiology Department, Heart Institute "Niculae Stăncioiu", ${ }^{3}$ Cardiovascular Surgery Department, Heart Institute "Niculae Stăncioiu”, Cluj- Napoca, Romania

\begin{abstract}
Assessment of the left internal mammary artery (LIMA) graft patency currently requires invasive investigation through coronary angiography. In the last years the successful application of noninvasive Doppler spectrum analysis has been reported for patency assessment of the LIMA graft after myocardial revascularization. Echocardiography is considered to be a sensitive noninvasive screening modality to diagnose critical narrowing of LIMA grafts and angiography should be reserved for cases in which Doppler echocardiography fails to visualize the LIMA or reveals an abnormal flow pattern.

Keywords: transthoracic echocardiography, color Doppler ultrasound, left internal mammary artery graft
\end{abstract}

\section{Introduction}

The left internal mammary artery (LIMA) is the graft of choice to the left anterior descending coronary artery (LAD). Since its introduction in the 1960s, it has contributed to improved survival [1] and longer graft patency after coronary artery bypass graft (CABG) surgery. Invasive cardiac catheterization is the definitive method for assessment of LIMA graft patency in patients with recurrent angina, myocardial ischemia or infarction after CABG. Transthoracic Doppler echocardiography (TDE) was first described by Gould [2] in 1972 as a noninvasive study of venous grafts. It was developed as an alternative to coronary angiography but was not widely used due to suboptimal sensitivity and specificity reports. Sometimes TDE techniques are limited by transducer positioning, vessel curvature and constant motion of the heart. The successful application of noninvasive Doppler spectrum analysis has been reported for the hemodynamic assess-

Received 15.08.2012 Accepted 25.09.2012

Med Ultrason

2013, Vol. 15, No 1, 45-50

Corresponding author: Pârv Andreea Amalia

7 Eugen Ionesco street

Cluj- Napoca, Romania

Phone: +40747 646877

Email: andreea.parv@yahoo.com ment of LIMA graft [3]. In this paper the normal anatomy of the LIMA, the technique for transthoracic Doppler sonography and the pulsed-wave Doppler derived parameters for assessing the graft patency will be discussed.

\section{Anatomy of the left internal mammary artery}

Knowledge of the normal anatomy of the LIMA is pertinent to interpreting postoperative bypass studies. The LIMA arises from the undersurface of the first portion of the subclavian artery opposite to the thyrocervical trunk. It descends behind the cartilages of the upper six ribs at a distance of about $1.25-1.5 \mathrm{~cm}$ from the margin of the sternum. In $95 \%$ of the cadavers the LIMA descends antero-medially behind the internal jugular vein and the brachiocephalic vein. Passing behind the first costal cartilage, close to the phrenic nerve, it goes down into the thorax. The LIMA is separated from the pleura at the second or third costal cartilage by a strong layer of endothoracic fascia and inferiorly by the transversus thoracic muscle. It is accompanied by two mammary veins, which ascend medially and laterally, respectively $[4,5]$. At the level of the sixth intercostal space it divides into the musculophrenic and superiorepigastric arteries. Its branches include the pericardiophrenic, anterior mediastinal, pericardial, sternal, intercostal, perfo- 
rating, musculophrenic, and superior epigastric arteries. The length of the LIMA varies from 15.1 to $26.0 \mathrm{~cm}$, with a mean of $20.4 \pm 2.1 \mathrm{~cm}$ from the origin to the termination point [6]. The LIMA is generally harvested as the pedicle that includes the veins, the muscle around it, and the fascia. The harvesting as a skeletonized conduit is becoming more and more popular, as it increases the length of the conduit.

\section{Coronary circulation physiopathology and Dop- pler flow characteristics}

The pulsatile aspect of coronary blood flow has been recognized since the 17th century [7]. Normal arterial Doppler signals are usually biphasic or triphasic [8]. The first component corresponds to the high velocity forward flow, which occurs during systole, that is, peak systolic velocity (PSV). The second is of a lower frequency than the first one and corresponds to reversed flow in early diastole, that is, end- diastolic velocity (EDV). This component is dependent on peripheral resistance and will be absent where peripheral resistance is low, as is the case of the coronary arteries. The third component of the pulsed wave Doppler signal is of a lower frequency than the first two components and represents forward flow in late diastole (PDV). Normal coronary blood flow as well as velocity pattern are biphasic, and the diastolic component predominates over the systolic component [9]. Non-invasive evaluation of coronary blood flow has confirmed the biphasic pattern of these arteries [10] (fig 1).

Flow in the LIMA has been investigated using TDE since the 1990s $[11,12]$. Blood flow in ungrafted LIMA occurs mainly during systole similar to the flow in peripheral arteries, like in the subclavian artery (fig 2). Using Doppler assessment, in situ LIMA is mainly perfused during systole [13] and displays a high resistance flow velocity pattern (strong systolic peak, negative or absent protodiastolic velocity and low diastolic peak), as was shown in the study by Cartier et al [13]. Once grafted to the left coronary network, the LIMA flow pattern instantly adapts to the left ventricular hemodynamic. The Doppler shift frequency drops during the systolic phase and becomes more prominent during the diastolic phase as the left ventricular and wall tension decreases. The diastolic flow velocity of the LIMA increases after CABG as the result of the physiologically decreased resistance in the coronary circulation. This "diastolisation" of the LIMA blood flow is also related to the low resistance and large capacitance of the coronary artery network and to the LIMA self-regulated property on vascular tone.

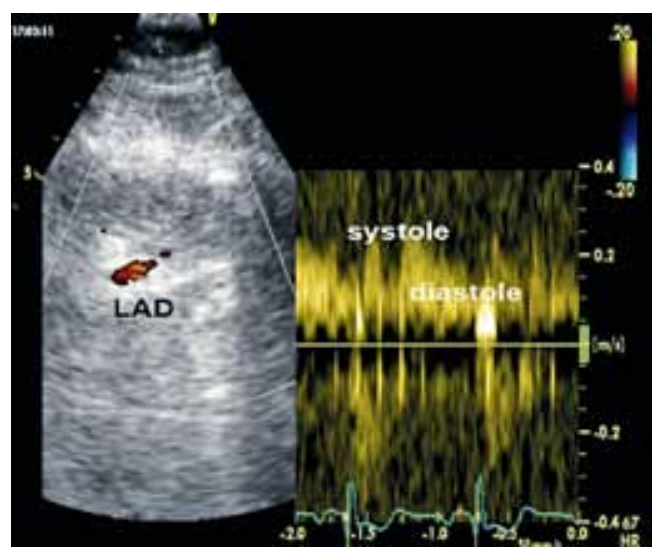

Fig 1. Biphasic Pulsed- wave color Doppler flow in the LAD

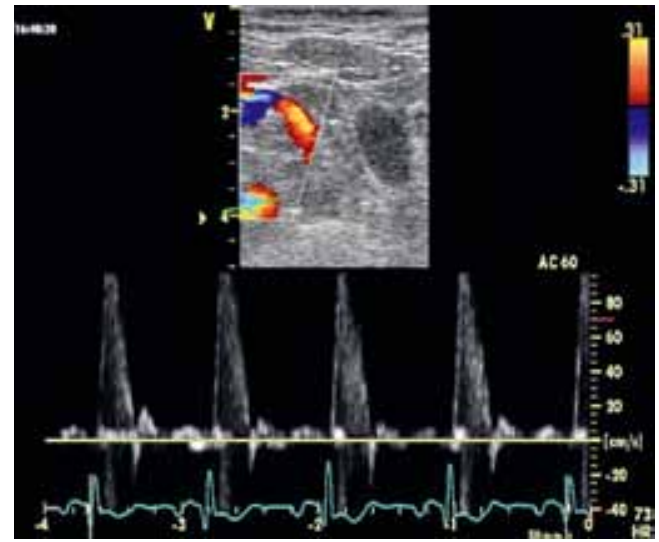

Fig 2. Triphasic Pulsed-wave color Doppler flow in the subclavin artery

\section{Pulsed wave and color Doppler assessment of the LIMA graft}

With the patient lying in supine position, a standard two-dimensional and Doppler echocardiography with 2-4 MHz transducer should be used. The LIMA graft can be imaged either from the left supraclavicular fossa [14] or from the left parasternal window of the intercostal spaces [11]. As the native course of the LIMA is more or less modified by the surgical procedure, the left supraclavicular fossa approach is preferred, which has the highest detection rate and is the best site for repeated scans. By TDE the LIMA rate detection is high, from $81 \%$ to $98 \%$ [3,15-17].

Supraclavicular window. To detect the LIMA flow, the initial velocity range of the color Doppler should be set at approximately $21 \mathrm{~cm} / \mathrm{sec}$ and subsequently adjusted to optimize visualization of color signals in the 


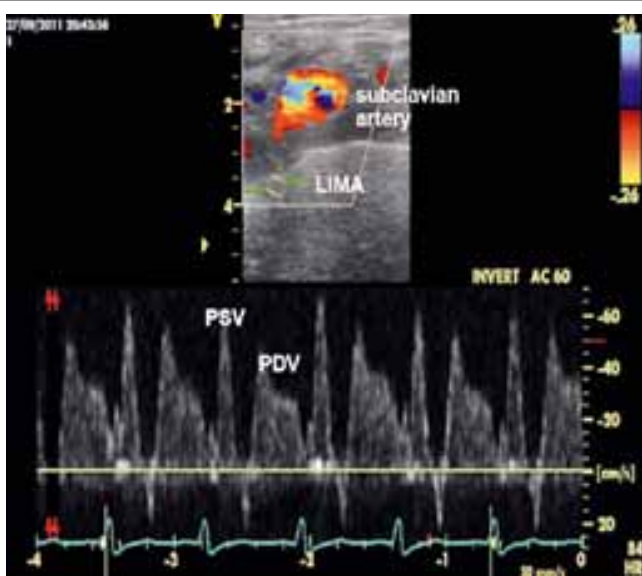

Fig 3. LIMA Pulsed-wave color Doppler flow aspect from the supraclavicular window, near its origin from the subclavian artery

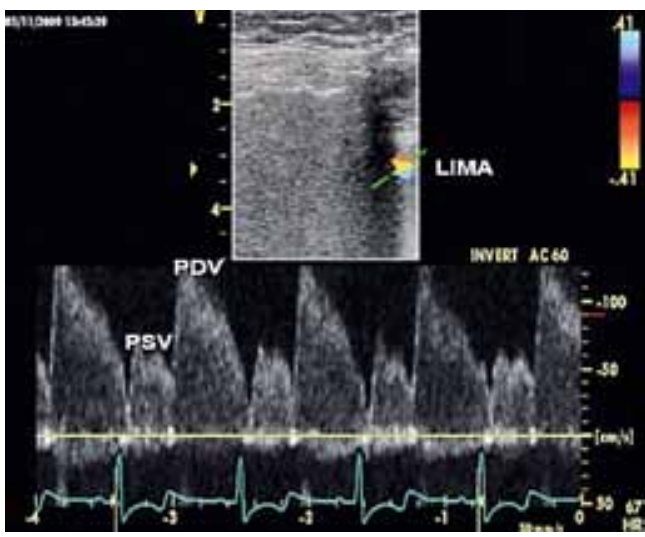

Fig 4. LIMA Pulsed-wave color Doppler flow aspect from the left parasternal window, with its peak systolic (PSV) and diastolic (PDV) velocities

bypass graft [18]. A transducer is placed on the left supraclavicular fossa, and the first task in detecting LIMA flow is to identify the left subclavian artery (SCA). The proximal LIMA is detected by rotating the transducer slightly clockwise and inclining it toward the anterior chest wall. Flow in LIMA is determined by recognizing downward flow in the tubular structure branching from the SCA. Flow velocity in the LIMA is examined 1.5 to $2.0 \mathrm{~cm}$ away from the SCA by pulsed-wave Doppler echocardiography with a sample volume of $2 \mathrm{~mm}$. The sample volume direction must be adjusted along the long axis of the LIMA flow for angle correction to determine flow velocity more accurately. The LIMA flow has a biphasic flow pattern by pulsed-wave Doppler (fig 3).
Left parasternal window. Several investigators have used the parasternal approach to measure flow in the distal LIMA. The rate of detecting LIMA flow is lower via the parasternal approach than via the supraclavicular approach $[3,19]$. According to Dubey et al [20] the LIMA is located in the second to fifth left intercostal space parasternally. Patients can be examined in the left lateral position or supine position, by using a modified left parasternal window. Long-axis images of the left ventricle must be obtained, and then the area anterior to the right ventricular outflow tract and the anterior interventricular sulcus are carefully examined by using combined imaging and color-flow mapping. The LIMA graft is identified as a tubular structure with color flow directed from base to apex and containing characteristic Doppler flow signals. Once the position of the LIMA is identified, intraluminal flow signals are obtained using the pulsed- wave Doppler method [9]. The long-axis sections must be carefully adjusted to minimize the angle between the Doppler beam and the long axis of the artery as well as to ensure that the sampling volume is located within the vessel lumen for as much of the cardiac cycle as possible (fig 4).

\section{Doppler flow derived parameters to assess LIMA graft patency}

By means of TDE it is difficult to detect moderate stenosis $(50-70 \%)$ of the LIMA grafts, as flow does not significantly differ between intermediately stenosed and patent grafts [12]. The following pulsed-wave Doppler derived parameters are useful to detect severe stenosis $(>75 \%)$ of the LIMA grafts.

1. Diastolic/ Systolic Peak Wave Velocity Ratio (PDV/PSV)

The peak systolic (PSV) and diastolic velocity (PDV) and the mean systolic and diastolic velocity are measured by tracing the outer borderline of the systolic and diastolic Doppler velocity profiles, respectively (fig 5). Each parameter must be obtained by averaging measurements from three to five consecutive cardiac cycles, and the peak and mean PDV/PSV must be calculated. The PDV/PSV ratio is used because it is independent of the ultrasound beam angle of incidence that affects Doppler calculations. With the use of a Doppler flow wire, Bach et al [16] showed that in LIMA grafts without stenosis the ratio PDV/PSV increases from 0.6 proximally to 1.4 distally, and studies using TDE generated similar results $[21,22]$. A predominant baseline diastolic flow velocity, similar to the native coronary pattern, with a peak diastolic flow velocity (PDV) greater than peak systolic flow velocity (PSV), is typical of LIMA without stenosis [12]. On the other hand, a predominant PSV at baseline, previ- 
ously considered as a marker of LIMA graft dysfunction [23] may also be detected in other conditions as residual mammary artery side branches $[12,15,24]$ and partially occluded recipient artery distal to the graft anastomosis $[24,25]$. Several reports have shown that a decrease in $\mathrm{PDV} / \mathrm{PSV}<0.5$ or 0.6 is a powerful predictive parameter of LIMA graft stenosis [23]. In the study of Mizukami et al [18] a mean PDV/PSV ratio $<0.57$ predicted severe graft stenosis $(>75 \%)$ with a sensitivity, specificity, and accuracy of $88 \%, 91 \%$, and $91 \%$, respectively, in patients with LIMA grafts. Patients with significant stenosis of the LAD distal to the anastomosis of the LIMA were excluded in order to avoid the influence of native coronary artery stenosis on the LIMA flow.

2. Systolic/Diastolic Peak Wave Velocity Ratio (PSV/PDV)

In the study of Madaric et al [26] it was shown that a ratio $\mathrm{PSV} / \mathrm{PDV}<2$ yielded optimal accuracy to detect the absence of LIMA bypass dysfunction with a negative predictive value of $95 \%$. Its positive predictive value was only 52.4\%. Luise et al [27] found that the ratio PSV/ PDV is higher in a LIMA graft with patent collaterals (i.e. in mammary arteries harvested without the use of specific devices through a minithoracotomy). Crowley et al [3] reported that at baseline a ratio PSV/ PDV > 1 in LIMA anastomosed to LAD predicted severe stenosis with a sensitivity of $100 \%$ and a specificity of $85 \%$ on TDE.

\section{Diastolic Fraction (DF)}

In the cardiovascular system, which is a pulsatile one, the velocity of the blood flow is not constant in time, that is why it must be taken into account all values of the instantaneous velocities of the flow through an orifice in a certain time, which is the velocity time integral (VTI). The VTI represents the equivalent of the area under the pulsed-wave Doppler envelope [12], and is measured in the following way:

$$
\mathrm{VTI}=\sum_{i=1}^{n} V i \times T i
$$

where Vi is the velocity at the time Ti. The VTI can be computed for both systolic (SVTI) and diastolic (DVTI) flow. The diastolic fraction (DF) is computed in the following manner (fig 6):

$$
D F=\frac{D V T I}{D V T I+S V T I} .
$$

Recently, it was proposed that a $\mathrm{DF}<50 \%$ predicts $>$ $70 \%$ stenosis in LIMA grafts with a sensitivity and specificity of $100 \%$ [28]. The DF is thought to be the most powerful predictor because the peak velocity ratio is affected by native vessel stenosis, and the diameter and

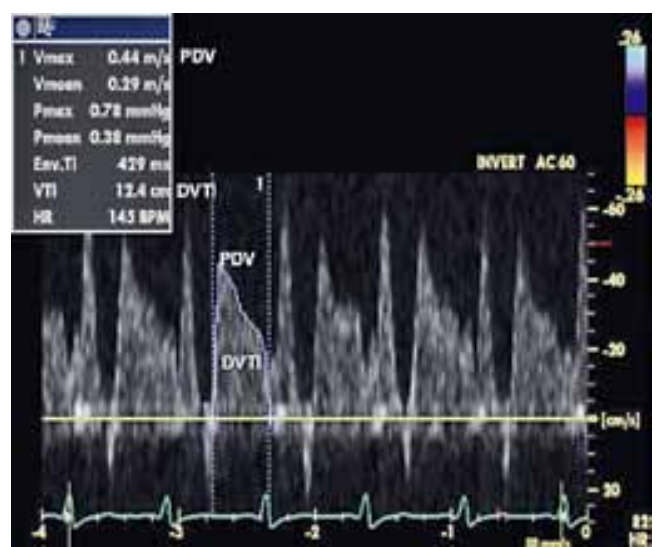

Fig 5. Method of computing the time velocity integral, here the diastolic time velocity integral (DVTI)

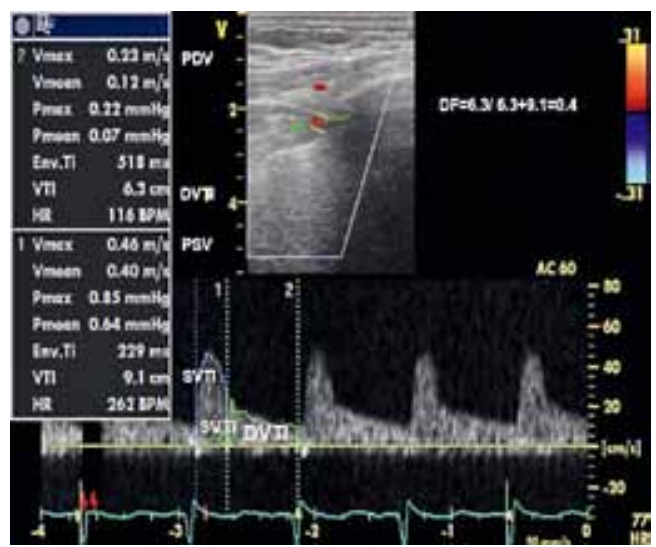

Fig 6. The method of computing the diastolic fraction (DF), according to the formula that uses the systolic (SVTI) and diastolic (DVTI) time velocity integrals

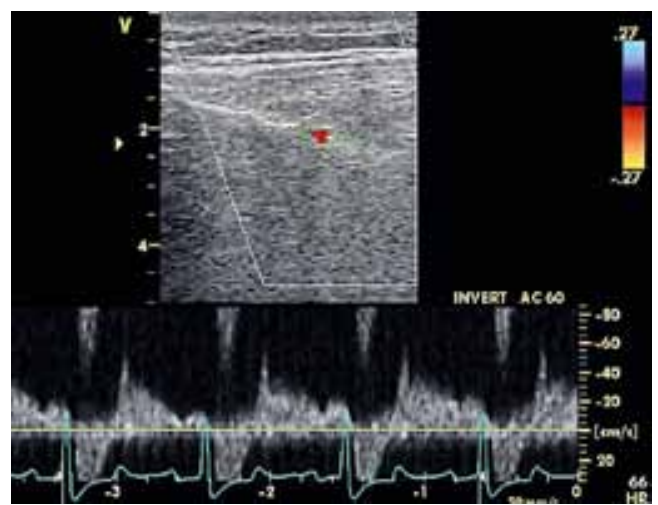

Fig 7. LIMA Pulsed-wave color Doppler flow in the 3 rd left intercostals space with systolic reversal flow and antegrade diastolic flow 
flow rate are influenced by the same factors [23]. Jones et al [17] described in their review report that the DF of less than $50 \%$ was shown to be the best criterion for the prediction of significant LIMA graft stenosis.

\section{Systolic reversal flow}

The study by Akasaka et al [29] in 1991 was the first study to describe the pattern in angiographically no-flow LIMA grafts. In no-flow patent grafts, a gradual longitudinal transition in the systolic flow velocity from antegrade proximally to retrograde distally was demonstrated, with lower antegrade diastolic velocity. To and fro signals with systolic reversal and diastolic antegrade flow (fig 7) were observed in the distal portion of the bypass grafts at baseline in the above mentioned study. The observed to and fro signals in the LIMAs graft might be the result of flow competition between the native coronary artery, when its stenosis before anastomosis is not severe, and the graft itself.

\section{Limitations and sources of error}

There are several limitations of the method: LIMA small size diameter, continuing moving of the heart, time consuming technique, and the need for digital ultrasound technology with dedicated machines. Therefore, for the technique to be of any clinical value, one would need very highly trained echocardiographers [25].

Important sources of error are the insufficient examiner's experience and insufficient time for examination, inappropriate use of some parameters (velocity scale, Doppler angle, placement and size of the sample volume, color and Doppler gain), and difficulties in interpreting LIMA graft with pathological Doppler signal, which may be easy mistaken with some large intercostals arteries.

\section{Conclusions}

TDE is a non-invasive, inexpensive, fast method, with a low risk of complications, in detecting the LIMA grafts. It can be performed both immediately and in the late postoperative period. Whilst it can be performed at the bedside or in the outpatient setting, it requires training in performing and interpreting results. Highly skilled operators are required to prevent mistakes in diagnosis.

\section{Conflict of interest: none}

\section{Bibliography:}

1. Sabik JF 3rd, Lytle BW, Blackstone EH, Houghtaling PL, Cosgrove DM. Comparison of saphenous vein and internal thoracic artery graft patency by coronary system. Ann Thorac Surg 2005; 79: 544-551.

2. Gould KL, Mozersky DJ, Hokanson DE, et al. A noninvasive technic for determining patency of saphenous vein coronary bypass grafts. Circulation 1972; 46: 595-600.

3. Crowley JJ, Shapiro LM. Noninvasive assessment of left internal mammary artery graft patency using transthoracic echocardiography. Circulation 1995; 92(9 Suppl): II25-30.

4. Buxton B, Norsworthy C, Eizenberg N, Liu JJ. Internal thoracic artery. In: Buxton B, Frazier OH, Westaby S. (editors). Ischemic Heart Disease Surgical Management, Mosby International, London 1999: 141-151.

5. Calafiore AM, Contini M, Iacò AL, et al. Angiographic anatomy of the grafted left internal mammary artery. Ann Thorac Surg 1999; 68: 1636-1639.

6. Henriquez-Pino JA, Gomes WJ, Prates JC, Buffolo E. Surgical anatomy of the internal thoracic artery. Ann Thorac Surg 1997; 64: 1041-1045.

7. Scaramucci J. De motu cordis, theorema sexton, Theoremata Familiara de physico-medicics lucubrationibus Iucta leges mechanicas. 1695: 70-81.

8. Canver CC, Armstrong VM, Nichols RD, Mentzer RM. Color-flow duplex ultrasound assessment of internal thoracic artery graft after coronary bypass. Ann Thorac Surg 1995; 59: 389-392.

9. El-Masry MM, Salama MM, Darwish AZ, Abd El- Aziz O. Assessment of left internal mammary artery graft patency by transthoracic Doppler echocardiography. Clin Cardiol 2002; $25: 511-516$.

10. Fusejima K. Noninvasive measurement of coronary artery blood flow using combined two-dimensional and Doppler echocardiography. J Am Coll Cardiol 1987; 10: 1024-1031.

11. Katz WE, Zenati M, Mandarino WA, Cohen HA, Gorcsan J 3rd. Assessment of left internal mammary artery graft patency and flow reserve after minimally invasive direct coronary artery bypass. Am J Cardiol 1999; 84: 795-801.

12. Hirata N, Asaoka N, Amemiya A, Hatsuoka S, Ueno T, Kosakai $\mathrm{Y}$. Noninvasive evaluation of internal thoracic artery and left anterior descending coronary artery anastomotic sites using transthoracic Doppler echocardiography: comparison with coronary arteriography. J Thorac Cardiovasc Surg 2003; 126: 1080-1086.

13. Cartier R, Dias OS, Pellerin M, Hebert Y, Leclerc Y. Changing flow pattern of the internal thoracic artery undergoing coronary bypass grafting - continuous-wave Doppler assessment. J Thorac Cardiovasc Surg 1Voudris V, Athanassopoulos G, Vassilikos V, et al. Usefulness of flow reserve in the left internal mammary artery to determine graft patency to the left anterior descending coronary artery. Amer J Cardiol 1999; 83: 1157-1163.

14. Kern MJ, Bach RG, Donohue TJ, Caracciolo EA, Wolford T, Aguirre FV,. Interventional physiology. Part XIII: Role of large pectoralis branch artery in flow through a patent left internal mammary artery conduit. Cathet Cardiovasc Diagn 1995; 34: 240-244.

15. Bach RG, Kern MJ, Donohue TJ, Aguirre FV, Caracciolo EA. Comparison of phasic blood flow velocity characteris- 
tics of arterial and venous coronary artery bypass conduits. Circulation 1993; 88: II 133-140.

16. Jones CM, Athanasiou T, Tekkis PP, et al. Does Doppler echography have a diagnostic role in patency assessment of internal thoracic artery grafts? Eur J Cardiothorac Surg 2005; 28: 692-700.

17. Mizukami N, Kisanuki A, Hamasaki S, et al. Different flow patterns between left and right internal thoracic artery grafts influence the evaluation of severe graft stenosis by transthoracic Doppler echocardiography. J Am Soc Echocardiogr 2011; 24: 768-774.

18. Kyo S, Matsumura M, Yokote Y, Takamoto S, Omoto R. Evaluation of patency of internal mammary artery grafts: a comparison of two-dimensional Doppler echocardiography with coronary angiography. J Cardiol 1990; 20: 607-616.

19. Dubey B, Bhan A, Choudhary SC, et al. Assessment of Internal Mammary Artery Graft Patency: Angiography or Doppler? Asian Cardiovasc Thorac Ann 2000; 8: 325-329.

20. Izumi C, Takahashi S, Kurozumi K, et al. Usefulness of high-frequency transthoracic Doppler echocardiography in noninvasive diagnosis of the left internal thoracic artery graft stenosis at the anastomosis. Circ J 2004; 68: 845-849.

21. Pizzuto F, Voci P, Sinatra R, Sardella G, Nigri A. Non-invasive assessment of coronary flow velocity reserve before and after angioplasty in a patient with mammary graft stenosis. Ital Heart J 2000; 1: 636-639.
22. Gurne O, Chenu P, Buche M, et al. Adaptive mechanisms of arterial and venous coronary bypass grafts to an increase in flow demand. Heart 1999; 82: 336-342.

23. Gregg DE, Sabiston DC Jr. Effect of cardiac contraction on coronary blood flow. Circulation 1957; 15: 14-20.

24. Chirillo F, Bruni A, De Leo A, et al. Usefulness of dipyridamole stress echocardiography for predicting graft patency after coronary artery bypass grafting. Am J Cardiol 2004; 93: 24-30.

25. Madaric J, Mistrik A, Riecansky I, et al. Left internal mammary artery bypass dysfunction after revascularization of moderately narrowed coronary lesions. Colour-duplex ultrasound versus angiography study. Eur J Echocardiogr 2008; 9: 273-277.

26. Luise R, Teodori G, Di Gianmarco G, et al. Persistence of mammary artery branches and blood supply to the left anterior descending. Ann Thorac Surg 1997; 63: 1759-1764.

27. Horneffer PJ, Gardner TJ, Manolio TA, et al. The effects of age on outcome after coronary bypass surgery. Circulation 1987; 76: V6-12.

28. Akasaka T, Yoshida K, Hozumi T, et al. Flow dynamics of angiographically no-flow patent internal mammary artery grafts. J Am Coll Cardiol 1998; 31: 1049-1056.

29. Pezzano A, Fusco R, Child M, et al. Assessment of left internal mammary artery grafts using dipyridamole Doppler echocardiography. Am J Cardiol 1997; 80: 1603-1606. 